\title{
Robust Observer for a Class of Nonlinear SISO Dynamical Systems
}

\author{
David Rosas, ${ }^{1}$ Joaquin Alvarez, ${ }^{2}$ Patricia Rosas, ${ }^{1}$ and Raul Rascon ${ }^{1}$ \\ ${ }^{1}$ Engineering Faculty, Autonomous University of Baja California, 21280 Mexicali, BC, Mexico \\ ${ }^{2}$ Applied Physics Division, CICESE Research Center, 22860 Ensenada, BC, Mexico \\ Correspondence should be addressed to David Rosas; drosas@uabc.edu.mx
}

Received 5 October 2015; Revised 7 February 2016; Accepted 9 February 2016

Academic Editor: Asier Ibeas

Copyright (c) 2016 David Rosas et al. This is an open access article distributed under the Creative Commons Attribution License, which permits unrestricted use, distribution, and reproduction in any medium, provided the original work is properly cited.

\begin{abstract}
A procedure to design an asymptotically stable second-order sliding mode observer for a class of single input single output (SISO) nonlinear systems in normal form is presented. The observer converges to the system state in spite of the existence of bounded disturbances and parameter uncertainties affecting the system dynamics. At the same time, the observer estimates the disturbances without the use of an additional filter to recover the equivalent control. The observer design is modular; each module of the observer is applied to each equation of state of the plant. Because of this, the proposed observer can be applied to a broader class of dynamic systems. The performance of the observer is illustrated in numerical and experimental form.
\end{abstract}

\section{Introduction}

A state observer is a system that provides an estimate of the state variables, parameters, and disturbances of a given real system, based on measurements of the inputs and outputs of that system. From the viewpoint of dynamic systems, the observer design problem arises from the need for information to carry out control, monitoring, and modeling. In this way, it is a major problem in implementing a control system in general $[1,2]$. Moreover, disturbances widely exist in modern control systems and bring about adverse effects to their performance. Therefore, disturbance rejection is one of the key objectives in controller design. The disturbances can arise not only from the external environment but from unmodeled dynamics, parameter perturbations, and nonlinear couplings of multivariable systems, which are difficult to handle [3]. Thus, a disturbance estimator can become a complement of a state observer, or even it can be included in the state observer design, as in our case.

In many cases, the design of observers depends largely on the model of the plant and on the knowledge of information of the nominal values on its parameters. These observers need an accurate model of the plant; if there are external disturbances or parameter uncertainties, the performance is diminished, causing stability problems in the closed loop system. Therefore, the problem of observation of systems with unknown inputs has been one of the most important problems in control theory during the last decades.

Currently, there are different observers that exhibit some degree of robustness and can be applied to linear and nonlinear systems. Because the observers design problem is related to the controllers design problem, different robust control techniques have been used to design robust observers; see, for example, [4-8]. Particularly, the observers based on sliding mode technique have shown good convergence properties and exhibit good performance to external bounded disturbances. For this reason, observers based on sliding modes are widely used because of their features such as robustness to unknown inputs and the possibility of using the equivalent control for the estimation of unknown inputs and obtaining convergence in finite time; see, for example, $[1,4,9,10]$. In what follows, some of the most important works on observers using the sliding mode technique are described.

An observer for Lipschitz nonlinear systems, with not only unknown inputs but also measurement noise when the observer matching condition is not satisfied, is presented 
in [11]. An important condition is that the disturbances in the state equation and the noise in the output must have a bounded derivative. Also, the total operation of the observer depends on the accuracy of the estimation of internal auxiliary variables on finite time, which theoretically can be done. However, in practice, there can be many problems due to nonidealities in physical systems which generate real sliding modes that produce chattering in the state.

In [12], a differentiator with finite time convergence based on the supertwisting algorithm is presented. This differentiator solves, in theory, the problem of the estimation of the state variables in systems that can be brought to normal form, but it cannot estimate disturbances in the systems.

Other important proposals are [7, 13]. Reference [13] presents an observer based on the supertwisting algorithm for mechanical systems with bounded disturbances. This observer ensures convergence in finite time to the state of the plant. In [7], based on second-order sliding modes technique, a robust observer for mechanical systems with uncertainties is presented. This observer has convergence in exponential form to the state of the plant. In both cases $[7,13]$, the equivalent control is equal to the disturbance terms in the plant and they can be recovered using a lowpass filter [14]. In [15], this principle was successfully used to compensate disturbances and give robustness in a closed loop control system; however, these observers can only be applied to systems with Lagrangian structure and there is not a procedure to tune the time constant in the low-pass filter to recover the perturbations.

Reference [8] presents an observer for a special class of nonlinear systems with bounded state. The observer can estimate the disturbances in the system through the equivalent control, but it needs a low-pass filter to recover the disturbance, which generates an error in the estimation. Finally, in [16], multiple cascading observers are applied to uncertain linear systems to solve the problem of fault detection; this is an interesting proposal but it needs a filter for each observer.

In this paper, an observer for SISO nonlinear systems in normal form, with parameter uncertainties and external disturbances, is presented. The observer is based on the results presented in [7], but unlike the observer shown there, this observer guarantees asymptotic convergence to the state of the plant and to the disturbances; therefore, the use of an additional filter to recover the equivalent control is avoided; this constitutes the main contribution of the present approach. A restriction to be made in the observer design is that the time derivative of the disturbance is upper bounded by a known constant. The observer design is modular; each module of the observer is applied to each equation of state of the plant; because of this, the proposed observer can be applied to a broader class of dynamic systems. The performance of the observer is illustrated through a numerical simulation where the observer estimates the state variables of a chaotic system. The performance is also illustrated in experimental form; the observer is applied on a mechanical system to estimate the state variables and the disturbances, which are incorporated in a control system to compensate them and obtain a robust closed loop system, similar to a sliding control system, but with a smooth control input.

The organization of this paper is as follows. Section 2 provides the problem statement and presents some preliminary definitions. Section 3 shows the general structure of the observer and the stability proof. Its performance is illustrated in Section 4 through two examples: an observer design for a scaled Rössler system and an experimental observer application in a control system. Finally, Section 5 presents final comments and conclusions.

\section{Preliminary Definitions and Problem Statement}

Consider a nonlinear SISO system described by

$$
\begin{aligned}
& \dot{\xi}=\psi(\xi)+\eta(\xi) u+\sigma(\xi) v(t), \\
& y=h(\xi)
\end{aligned}
$$

where $\xi \in \mathfrak{R}^{n}$ is the state vector and $\psi: \mathfrak{R}^{n} \rightarrow \mathfrak{R}^{n}, \eta: \mathfrak{R}^{n} \rightarrow$ $\mathfrak{R}^{n}, \sigma: \mathfrak{R}^{n} \rightarrow \mathfrak{R}^{n}$, and $h: \mathfrak{R}^{n} \rightarrow \mathfrak{R}$ are functions $C^{k}$, for sufficiently large $k . u \in \mathfrak{R}$ is the control input and $v(t)$ is a bounded unknown external disturbance.

Consider that system (1) has relative degree $n$ with respect to output $y$, input $u$, and disturbance $v(t)$; then, there exists a set of transformations well defined in a subspace of the state space, such that system (1) can be rewritten as

$$
\begin{aligned}
& \dot{x}_{i}=x_{i+1}, \quad i=1, \ldots, n-1, \\
& \dot{x}_{n}=f(x)+g(x) u+\gamma(x) v(t), \\
& y=x_{1},
\end{aligned}
$$

where functions $f(\cdot), g(\cdot)$, and $\gamma(\cdot)$ are defined through Lie derivatives [17].

The problem is to design an observer for system (1) whose objective is to estimate the state $\xi$ and the disturbance $v(t)$. This problem is equivalent to designing an observer for system (2) and to obtaining $\xi$ using the inverse transformation.

\section{Observer Design for SISO Systems}

Consider that system (2) has bounded behavior for the input $u$ and the disturbance term $\gamma(x) v(t)$ and that $\left|x_{i}\right|<\delta_{i}$ and $\left|\dot{x}_{i}\right|<\delta_{i}^{\prime}$, for $i=1, \ldots, n$, and $|(d / d t) \gamma(x) v(t)|<\sigma$, where $\delta_{i}$, $\delta_{i}^{\prime}$, and $\sigma$ are known finite constants. 
The proposed observer has the form

$$
\begin{gathered}
\dot{\hat{x}}_{1}=c_{1,1}\left(y-\widehat{y}_{1}\right)+w_{1}, \\
\dot{w}_{1}=c_{2,1}\left(y-\widehat{y}_{1}\right)+c_{3,1} \operatorname{sign}\left(y-\widehat{y}_{1}\right), \\
\hat{y}_{1}=\widehat{x}_{1}, \\
\vdots \\
\dot{\hat{x}}_{i}=c_{1, i}\left(w_{i-1}-\widehat{y}_{i}\right)+w_{i}, \\
\dot{w}_{i}=c_{2, i}\left(w_{i-1}-\hat{y}_{i}\right)+c_{3, i} \operatorname{sign}\left(w_{i-1}-\widehat{y}_{i}\right), \\
\widehat{y}_{i}=\widehat{x}_{i}, \quad i=2, \ldots, n-1, \\
\vdots \\
\dot{\hat{x}}_{n}=f(y, \hat{x})+g(y, \hat{x}) u+c_{1, n}\left(w_{n-1}-\widehat{y}_{n}\right)+w_{n}, \\
\dot{w}_{n}=c_{2, n}\left(w_{n-1}-\widehat{y}_{n}\right)+c_{3, n} \operatorname{sign}\left(w_{n-1}-\widehat{y}_{n}\right), \\
\hat{y}_{n}=\widehat{x}_{n} .
\end{gathered}
$$

As we can see, for each state of system (2), there are two states and an output in observer (3). To analyze the stability of the observer, we define the error variables

$$
e_{i}=x_{i}-\widehat{x}_{i}, \quad i=1, \ldots, n,
$$

whose dynamics are given by the system

$$
\begin{aligned}
& \dot{e}_{1}=x_{2}-c_{1,1} e_{1}-w_{1}, \\
& \dot{w}_{1}=c_{2,1} e_{1}+c_{3,1} \operatorname{sign}\left(e_{1}\right), \\
& \dot{e}_{2}=x_{3}-c_{1,2}\left(w_{1}-x_{2}+e_{2}\right)-w_{2}, \\
& \dot{w}_{2}=c_{2,2}\left(w_{1}-x_{2}+e_{2}\right)+c_{3,2} \operatorname{sign}\left(w_{1}-x_{2}+e_{2}\right), \\
& \quad \vdots \\
& \quad \vdots \\
& \dot{e}_{i}=x_{i+1}-c_{1, i}\left(w_{i-1}-x_{i}+e_{i}\right)-w_{i}, \\
& \dot{w}_{i}=c_{2, i}\left(w_{i-1}-x_{i}+e_{i}\right)+c_{3, i} \operatorname{sign}\left(w_{i-1}-x_{i}+e_{i}\right), \\
& \dot{e}_{n}=f(x)+\gamma(x) v(t)-f(\widehat{x})-c_{1, n}\left(w_{n-1}-x_{n}+e_{n}\right) \\
& -w_{n}, \\
& \dot{w}_{n}=c_{2, n}\left(w_{n-1}-x_{n}+e_{n}\right)+c_{3, n} \operatorname{sign}\left(w_{n-1}-x_{n}+e_{n}\right) .
\end{aligned}
$$

The stability analysis of system (5) is made in several steps. It is important to note that the behavior of variables $e_{1}$ and $w_{1}$ is independent of the rest of the state variables of system (5); the behavior of variables $e_{2}$ and $w_{2}$ depends on variables $e_{1}$ and $w_{1}$ but not on the rest of the state variables, and so on in each block.
Take the first two equations of system (5):

$$
\begin{aligned}
& \dot{e}_{1}=x_{2}-c_{1,1} e_{1}-w_{1}, \\
& \dot{w}_{1}=c_{2,1} e_{1}+c_{3,1} \operatorname{sign}\left(e_{1}\right) ;
\end{aligned}
$$

because the behavior of the plant is considered bounded, we have that $\left|x_{2}\right|<\delta_{2}$ and $\left|\dot{x}_{2}\right|<\delta_{2}^{\prime}$. Making the change of variables $z_{1,1}=e_{1}$ and $z_{2,1}=x_{2}-c_{1,1} e_{1}-w_{1}$, system $(6)$ can be rewritten as

$$
\begin{aligned}
& \dot{z}_{1,1}=z_{2,1}, \\
& \dot{z}_{2,1}=\dot{x}_{2}-c_{1,1} z_{2,1}-c_{2,1} z_{1,1}-c_{3,1} \operatorname{sign}\left(z_{1,1}\right) ;
\end{aligned}
$$

define matrix $A$ as

$$
A=\left[\begin{array}{cc}
0 & 1 \\
-c_{2,1} & -c_{1,1}
\end{array}\right],
$$

and matrix $P$, which is the solution of the Lyapunov equation $A^{T} P+P A=-I$, for matrix $A$, as

$$
P=\left[\begin{array}{ll}
p_{11} & p_{12} \\
p_{21} & p_{22}
\end{array}\right]
$$

the stability properties of system (7) are given by the following theorem.

Theorem 1. For system (7), if

$$
c_{3,1}>2 \lambda_{\max }(P) \sqrt{\frac{\lambda_{\max }(P)}{\lambda_{\min }(P)}}\left(\frac{c_{2,1} \delta_{2}^{\prime}}{\theta}\right),
$$

for some $0<\theta<1$, then the origin of the state space is a globally asymptotically stable equilibrium in the Lyapunov sense.

Proof. The proof of this theorem is in [7].

Therefore, a set of constants $c_{1,1}, c_{2,1}$, and $c_{3,1}$ can be found such that the origin of the system is asymptotically stable; then, $w_{1}$ converges to $x_{2}$ and $\hat{x}_{1}$ to $x_{1}$.

Now, the dynamics of variables $e_{2}$ and $w_{2}$ are given by

$$
\begin{aligned}
& \dot{e}_{2}=x_{3}-c_{1,2}\left(w_{1}-x_{2}+e_{2}\right)-w_{2}, \\
& \dot{w}_{2}=c_{2,2}\left(w_{1}-x_{2}+e_{2}\right)+c_{3,2} \operatorname{sign}\left(w_{1}-x_{2}+e_{2}\right) ;
\end{aligned}
$$

defining a new set of variables

$$
\begin{aligned}
& z_{1,2}=w_{1}-x_{2}+e_{2}, \\
& z_{2,2}=\dot{z}_{1,2}
\end{aligned}
$$

whose dynamics are given by

$$
\begin{aligned}
& \dot{z}_{1,2}=z_{2,2} \\
& \dot{z}_{2,2}=\ddot{w}_{1}-\ddot{x}_{2}+\dot{x}_{3}-c_{1,2} z_{2,2}-\dot{w}_{2}
\end{aligned}
$$


and substituting $\dot{w}_{2}$, we obtain

$$
\begin{aligned}
\dot{z}_{1,2}= & z_{2,2}, \\
\dot{z}_{2,2}= & \ddot{w}_{1}-\ddot{x}_{2}+\dot{x}_{3}-c_{1,2} z_{2,2}-c_{2,2} z_{1,2} \\
& -c_{3,2} \operatorname{sign}\left(z_{1,2}\right) .
\end{aligned}
$$

It is important to note that the term $\ddot{w}_{1}-\ddot{x}_{2}$ goes to zero due to the first stage of the observer and that $\left|\dot{x}_{3}\right|<\delta_{3}^{\prime}$; for this reason and using Theorem 1 , a set of constants $c_{1,2}, c_{2,2}$, and $c_{3,2}$ can be found such that the origin of system (14) is an exponentially stable equilibrium point; therefore, $\hat{x}_{2}$ converges to $x_{2}$ and $w_{2}$ converges to $x_{3}$.

Now, to analyze block $i$, consider equations

$$
\begin{aligned}
& \dot{e}_{i}=x_{i+1}-c_{1, i}\left(w_{i-1}-x_{i}+e_{i}\right)-w_{i}, \\
& \dot{w}_{i}=c_{2, i}\left(w_{i-1}-x_{i}+e_{i}\right)+c_{3, i} \operatorname{sign}\left(w_{i-1}-x_{i}+e_{i}\right)
\end{aligned}
$$

and a set of variables

$$
\begin{aligned}
& z_{1, i}=w_{i-1}-x_{i}+e_{i}, \\
& z_{2, i}=\dot{z}_{1, i},
\end{aligned}
$$

whose dynamics are given by the system

$$
\begin{aligned}
\dot{z}_{1, i}= & z_{2, i}, \\
\dot{z}_{2, i}= & \ddot{w}_{i-1}-\ddot{x}_{i}+\dot{x}_{i+1}-c_{1, i} z_{2, i}-c_{2, i} z_{1, i} \\
& \quad-c_{3, i} \operatorname{sign}\left(z_{1, i}\right) .
\end{aligned}
$$

In this case, $\ddot{w}_{i-1}-\ddot{x}_{i}$ goes to zero due to the previous stage of the observer and $\left|\dot{x}_{i+1}\right|<\delta_{i+1}^{\prime}$. Using Theorem 1, we can find constants $c_{1, i}, c_{2, i}$, and $c_{3, i}$ such that the origin of system (17) is an exponentially stable equilibrium point; therefore, $\widehat{x}_{i}$ converges to $x_{i}$ and $w_{i}$ converges to $x_{i+1}$.

Finally, we analyze block $n$. Consider the equations

$$
\begin{aligned}
\dot{e}_{n}= & f(x)+\gamma(x) v(t)-f(\widehat{x})-c_{1, n}\left(w_{n-1}-x_{n}+e_{n}\right) \\
& -w_{n}, \\
\dot{w}_{n}= & c_{2, n}\left(w_{n-1}-x_{n}+e_{n}\right) \\
& +c_{3, n} \operatorname{sign}\left(w_{n-1}-x_{n}+e_{n}\right) ;
\end{aligned}
$$

for simplicity, a function $\phi(\cdot)$ is defined as

$$
\phi(\cdot)=f(x)+\gamma(x) v(t)-f(\widehat{x}),
$$

such that system (18) can be rewritten as

$$
\begin{aligned}
\dot{e}_{n}= & \phi(\cdot)-c_{1, n}\left(w_{n-1}-x_{n}+e_{n}\right)-w_{n} \\
\dot{w}_{n}= & c_{2, n}\left(w_{n-1}-x_{n}+e_{n}\right) \\
& +c_{3, n} \operatorname{sign}\left(w_{n-1}-x_{n}+e_{n}\right)
\end{aligned}
$$

we define a new set of variables

$$
\begin{aligned}
& z_{1, n}=w_{n-1}-x_{n}+e_{n}, \\
& z_{2, n}=\dot{z}_{1, n},
\end{aligned}
$$

whose dynamics are given by the system

$$
\begin{aligned}
\dot{z}_{1, n}= & z_{2, n}, \\
\dot{z}_{2, n}= & \ddot{w}_{n-1}-\ddot{x}_{n}+\dot{\phi}(\cdot)-c_{1, n} z_{2, n}-c_{2, n} z_{1, n} \\
& +c_{3, n} \operatorname{sign}\left(z_{1, n}\right) .
\end{aligned}
$$

Since $\ddot{w}_{n-1}-\ddot{x}_{n}$ goes to zero due to the previous stage of the observer and $\dot{\phi}(\cdot)$ is considered bounded because $(d / d t) \gamma(x) v(t)$ is bounded, applying Theorem 1 , a set of constants $c_{1, n}, c_{2, n}$, and $c_{3, n}$ can be found such that the origin of system (20) is an exponentially stable equilibrium point; therefore, $\widehat{x}_{n}$ converges to $x_{n}$ and $w_{n}$ converges to $\gamma(x) v(t)$.

It is important to note that the proposed observer, unlike others such as those presented in $[4,7]$, does not need to add low-pass filters to recover the disturbance terms.

\section{Observer Performance}

This section illustrates the performance of the observer through two examples. The first is a numerical simulation where the observer is used to estimate the state vector of a scaled version of the Rössler system under chaotic behavior.

The second example is an experiment where the observer is included in a control system; the observer estimates and compensates the velocities and disturbance terms due to parametric uncertainties and unmodeled dynamics in a mechanical system; thus, the closed loop system became robust.

4.1. Observer Design for a Scaled Rössler System. Consider a scaled version of the Rössler system modeled by

$$
\begin{aligned}
& \dot{x}_{1}=-x_{2}-x_{3}, \\
& \dot{x}_{2}=x_{1}+a x_{2}, \\
& \dot{x}_{3}=\frac{b}{\alpha}+x_{3}\left(x_{1} \alpha-c\right)+u, \\
& y=x_{2},
\end{aligned}
$$

where $a, b, c$, and $\alpha$ are constants, $u$ is a control input, and $y$ is the output. For $a=0.2, b=0.2, c=5.7$, and $\alpha>0$, the system shows a chaotic behavior; therefore, all state variables are bounded [18].

Using the transformation

$$
\begin{aligned}
& z_{1}=x_{2}, \\
& z_{2}=x_{1}+a x_{2}, \\
& z_{3}=-x_{2}-x_{3}+a z_{2},
\end{aligned}
$$

with inverse given by

$$
\begin{aligned}
& x_{1}=z_{2}-a z_{1}, \\
& x_{2}=z_{2}, \\
& x_{3}=-z_{1}+a z_{2}-z_{3},
\end{aligned}
$$


system (23) takes the normal form

$$
\begin{aligned}
\dot{z}_{1}= & z_{2}, \\
\dot{z}_{2}= & z_{3}, \\
\dot{z}_{3}= & -z_{2}-\frac{b}{\alpha}-\left(-z_{1}+a z_{2}-z_{3}\right)\left(\left(z_{2}-a z_{1}\right) \alpha-c\right) \\
& \quad+a z_{3}-u, \\
y= & z_{1} .
\end{aligned}
$$

The observer takes the following form:

$$
\begin{aligned}
& \dot{\hat{z}}_{1}=c_{1,1}\left(z_{1}-\widehat{z}_{1}\right)+w_{1}, \\
& \dot{w}_{1}=c_{2,1}\left(z_{1}-\widehat{z}_{1}\right)+c_{3,1} \operatorname{sign}\left(z_{1}-\widehat{z}_{1}\right), \\
& \widehat{y}_{1}=\widehat{x}_{1}, \\
& \dot{\hat{z}}_{2}=c_{1,2}\left(w_{1}-\widehat{z}_{2}\right)+w_{2}, \\
& \dot{w}_{2}=c_{2,2}\left(w_{1}-\widehat{z}_{2}\right)+c_{3,2} \operatorname{sign}\left(w_{1}-\widehat{z}_{2}\right), \\
& \widehat{y}_{2}=\widehat{z}_{2}, \\
& \dot{\vec{z}}_{3}=-u+c_{1,3}\left(w_{2}-\widehat{z}_{3}\right)+w_{3}, \\
& \dot{w}_{3}=c_{2,3}\left(w_{2}-\widehat{z}_{3}\right)+c_{3,3} \operatorname{sign}\left(w_{2}-\widehat{z}_{3}\right), \\
& \widehat{y}_{3}=\widehat{z}_{3} .
\end{aligned}
$$

It is important to note that the equation for $\dot{\hat{z}}_{3}$ does not include the term $-z_{2}-b / \alpha-\left(-z_{1}+a z_{2}-z_{3}\right)\left(\left(z_{2}-a z_{1}\right) \alpha-c\right)+$ $a z_{3}$, so this term is the disturbance $\gamma(z) v(t)$. The parameters of the observer are the following: $c_{1,1}=25, c_{2,1}=1, c_{3,1}=7$, $c_{1,2}=35, c_{2,2}=10, c_{3,2}=25, c_{1,3}=25, c_{2,3}=1$, and $c_{3,3}=55$.

Figure 1 shows the behavior of the actual and the observed state variables in $z$ space; dashed lines correspond to $z$ variables and continuous lines correspond to $\widehat{z}$ variables. Figure 1(a) corresponds to $z_{1}$ and $\widehat{z}_{1}$, Figure $1(\mathrm{~b})$ corresponds to $z_{2}$ and $\widehat{z}_{2}$, Figure $1(\mathrm{c})$ corresponds to $z_{3}$ and $\widehat{z}_{3}$, and Figures 1(d), 1(e), and 1(f) correspond to the errors between actual and observed variables. As we can see, the observer converges to the plant in a short time. In this simulation, the initial conditions in the plant were different to the initial conditions in the observer.

Figure 2, with the same order of Figure 1, shows the behavior of the actual and the observed state variables in $x$ space. It should be taken into account that observed state variables were obtained through the application of inverse transforms (26). Finally, Figure 3 shows the actual disturbance term $\gamma(z) v(t)$ and the disturbance estimated by $w_{3}$.

In practice, each stage of the observer has an actual sliding mode instead of an ideal sliding mode, which produces high frequency components with small amplitude in the observed variables; these components produce a cumulative effect. Because of this fact, a greater content of high frequency components (see Figure 3) is seen in the estimation of the disturbance, which is generated in the last stage of the observer.
4.2. Experimental Observer Application in a Control System. In this subsection, we use the proposed observer to estimate velocities and disturbances in a mechanical system; the estimated disturbances are used in the controller to compensate the real ones in the plant. As a result, we obtain a robust closed loop system, like those where a sliding mode controller is applied, but with a control signal free from high frequency components.

Consider the pendulum shown in Figure 4; its model is given by

$$
\begin{aligned}
& \dot{x}_{1}=x_{2}, \\
& \dot{x}_{2}=-76.34 \sin \left(x_{1}\right)-2.5 x_{2}+101.3 u+\gamma(\cdot), \\
& y=x_{1},
\end{aligned}
$$

where $x_{1}$ and $x_{2}$ are the angular position and velocity, respectively, $u$ is the control input, and $\gamma(\cdot)$ is a term that incorporates external disturbances and terms due to parametric uncertainties; we assume that both $\gamma(\cdot)$ and $\dot{\gamma}(\cdot)$ are bounded. The control objective is that the output $y$ tracks a reference signal $y_{\text {ref }}(t)$ :

$$
\lim _{t \rightarrow \infty}\left|y-y_{\text {ref }}(t)\right|=0
$$

4.2.1. The Control Structure. To design a controller, we define the error variables $\varepsilon_{1}=x_{1}-y_{\text {ref }}(t)$ and $\varepsilon_{2}=x_{2}-\dot{y}_{\text {ref }}(t)$, whose dynamics are given by

$$
\begin{aligned}
\dot{\varepsilon}_{1}= & \varepsilon_{2}, \\
\dot{\varepsilon}_{2}= & -76.34 \sin \left(x_{1}\right)-2.5\left(\varepsilon_{2}+\dot{y}_{\text {ref }}(t)\right)+101.3 u \\
& +\gamma(\cdot)-\ddot{y}_{\text {ref }}(t) .
\end{aligned}
$$

We propose a control input $u$ defined as $u=\tau+\sigma$, where $\tau$ is a nominal control and $\sigma$ is an auxiliary control. It is assumed that the nominal control produces a bounded behavior in the plant; in this example, we define $\tau$ as

$$
\begin{aligned}
\tau= & \frac{1}{101.3}\left(76.34 \sin \left(x_{1}\right)+2.5 \dot{y}_{\text {ref }}(t)-k_{p} \varepsilon_{1}\right. \\
& \left.+\ddot{y}_{\text {ref }}(t)\right) .
\end{aligned}
$$

Substituting (32) in (31) and considering the fact that $\gamma(\cdot)=0$, we have

$$
\begin{aligned}
& \dot{\varepsilon}_{1}=\varepsilon_{2}, \\
& \dot{\varepsilon}_{2}=\dot{\varepsilon}_{2}=-k_{p} \varepsilon_{1}-2.5 \varepsilon_{2} ;
\end{aligned}
$$

if the constant $k_{p}$ is positive, the origin of system (33) is an exponentially stable equilibrium point. If the uncertainty term $\gamma(\cdot)$ is small and bounded, the behavior of system (31) with control (32) is bounded too [17].

Now, we propose the additional term $\sigma$ as

$$
\sigma=\frac{1}{101.3}\left(-k_{v}\left(\hat{x}_{2}-\dot{y}_{\text {ref }}(t)\right)-\tilde{\gamma}(\cdot)\right)
$$




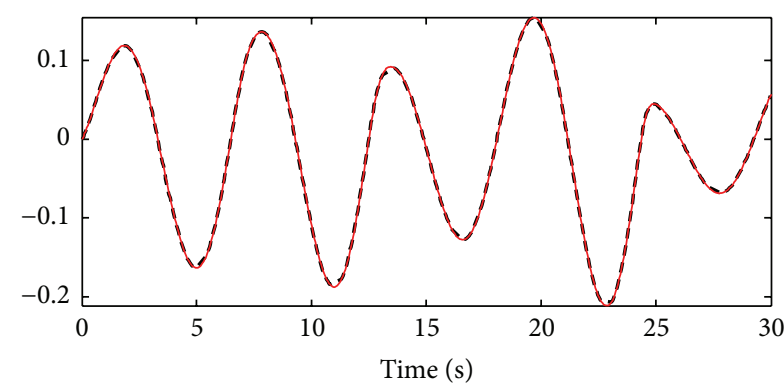

(a)

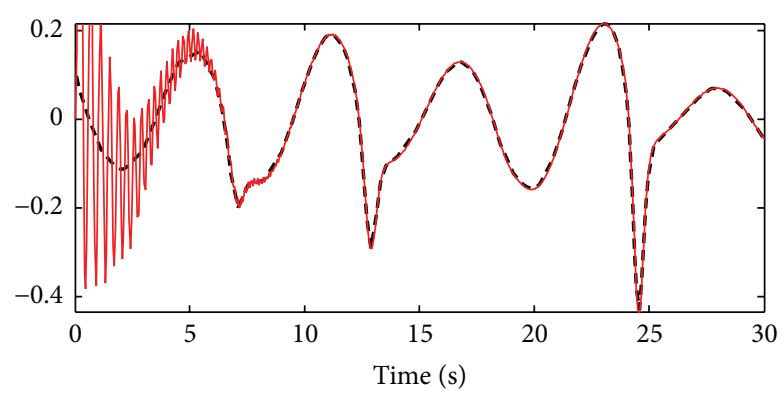

(c)

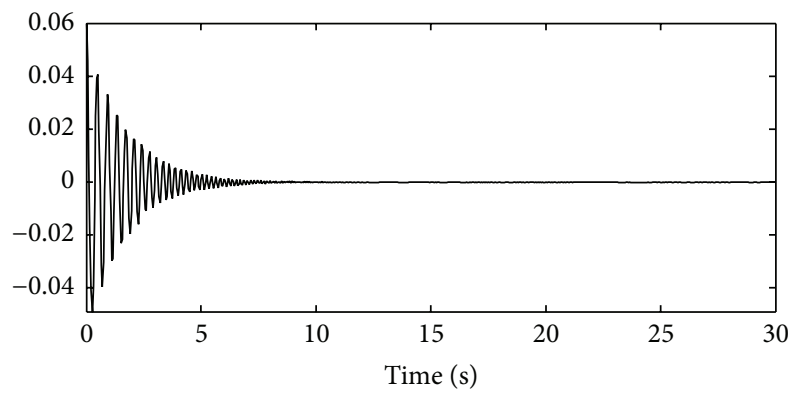

(e)

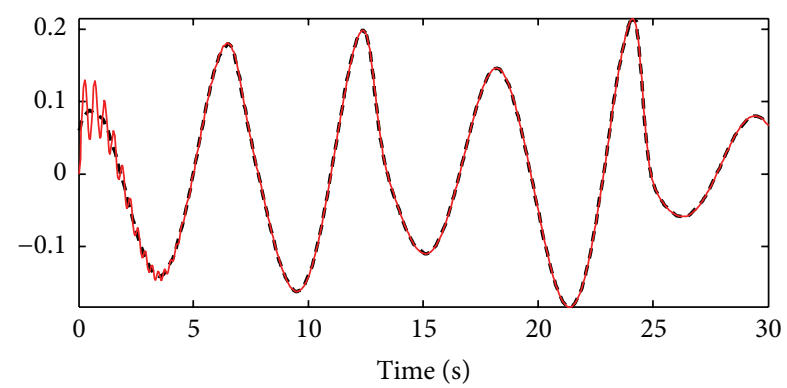

(b)

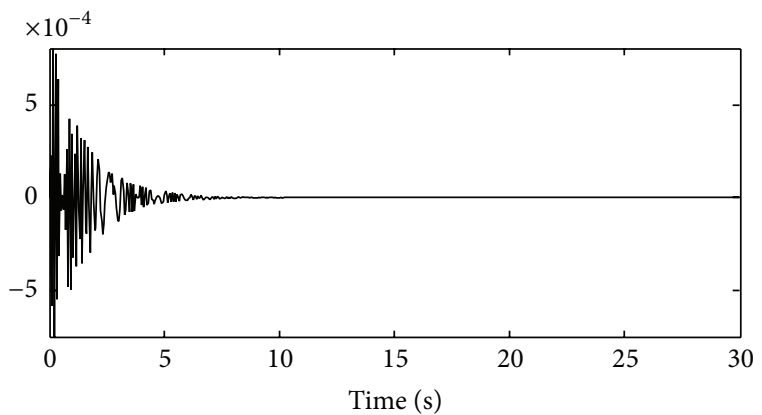

(d)

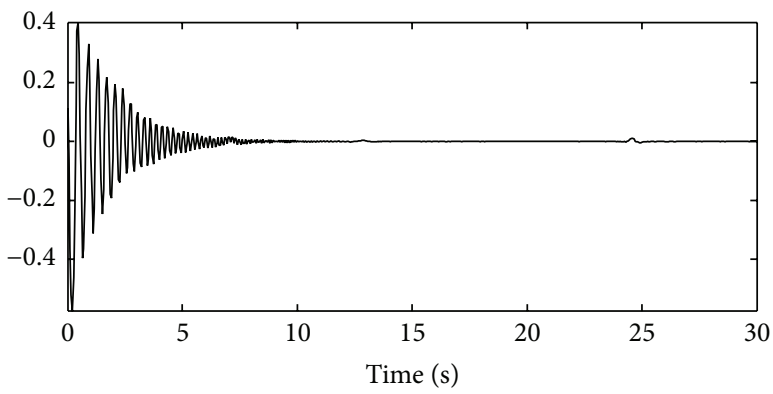

(f)

Figure 1: Behavior of the plant and the observer in $z$ space.

where $\widehat{x}_{2}$ and $\widetilde{\gamma}(\cdot)$ are estimated by an observer. Therefore, the full control signal takes the form

$$
\begin{aligned}
u= & \frac{1}{101.3}\left(76.34 \sin \left(x_{1}\right)+2.5 \dot{y}_{\text {ref }}(t)-k_{p} \varepsilon_{1}\right. \\
& \left.+\ddot{y}_{\text {ref }}(t)-k_{v} \widetilde{\varepsilon}_{2}-\widetilde{\gamma}(\cdot)\right),
\end{aligned}
$$

where $\widetilde{\varepsilon}_{2}=\widehat{x}_{2}-\dot{y}_{\text {ref }}(t)$. Substituting (35) in (31), we have

$$
\begin{aligned}
& \dot{\varepsilon}_{1}=\varepsilon_{2}, \\
& \dot{\varepsilon}_{2}=-k_{p} \varepsilon_{1}-k_{v} \widetilde{\varepsilon}_{2}-2.5 \varepsilon_{2}+\gamma(\cdot)-\widetilde{\gamma}(\cdot) .
\end{aligned}
$$

If an asymptotic observer is used such that the term $-k_{v} \Delta_{\varepsilon_{2}}+$ $\gamma(\cdot)-\widetilde{\gamma}(\cdot)$, where $\Delta_{\varepsilon_{2}}=\varepsilon_{2}-\widetilde{\varepsilon}_{2}$, goes to zero in asymptotic form, then the constants $k_{p}$ and $k_{v}$ may be chosen such that the origin of system (36) will be an asymptotically stable equilibrium point [17].
4.2.2. The Performance of the Observer. For the plant given by (29), the proposed observer is

$$
\begin{aligned}
\dot{\hat{x}}_{1}= & c_{11}\left(x_{1}-\hat{x}_{1}\right)+w_{1}, \\
\dot{w}_{1}= & c_{21}\left(x_{1}-\hat{x}_{1}\right)+c_{31} \operatorname{sign}\left(x_{1}-\hat{x}_{1}\right), \\
\hat{y}_{1}= & \widehat{x}_{1}, \\
\dot{\hat{x}}_{2}= & -76.34 \sin \left(x_{1}\right)-2.5 \hat{x}_{2}+101.3 u \\
& +c_{12}\left(w_{1}-\hat{x}_{2}\right)+w_{2}, \\
\dot{w}_{2}= & c_{22}\left(w_{1}-\widehat{x}_{2}\right)+c_{32} \operatorname{sign}\left(w_{1}-\widehat{x}_{2}\right), \\
\hat{y}_{2}= & \hat{x}_{2},
\end{aligned}
$$

where $c_{11}=50, c_{21}=80, c_{31}=20, c_{12}=50, c_{22}=300$, and $c_{32}=80$. The full control system was implemented on a 1103 dSPACE control platform, with a sample time of $1 \times 10^{-5}$ 


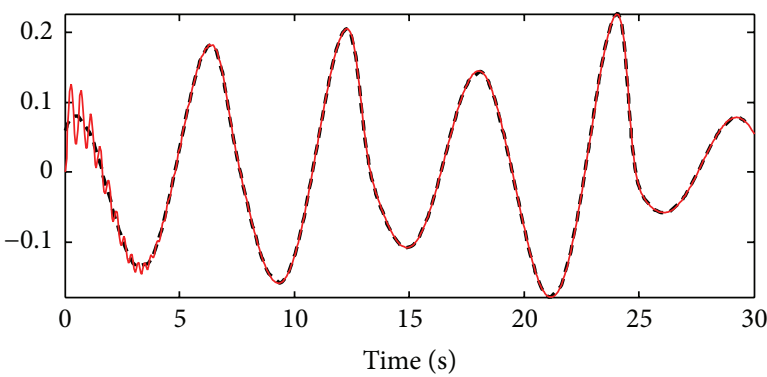

(a)

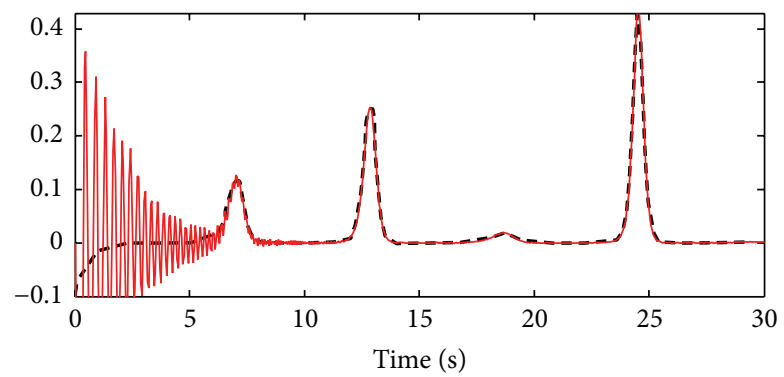

(c)

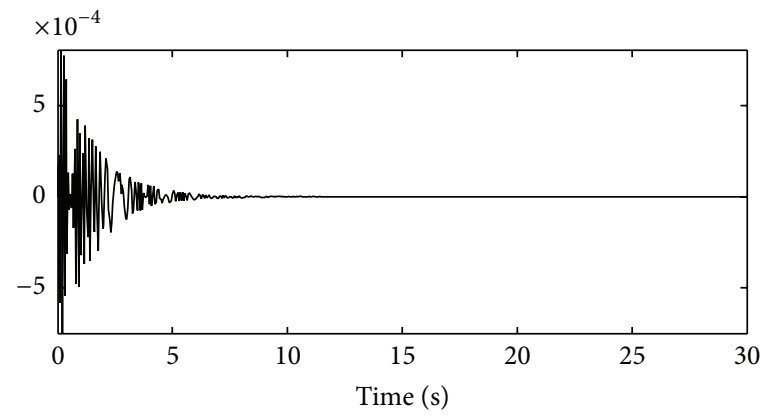

(e)

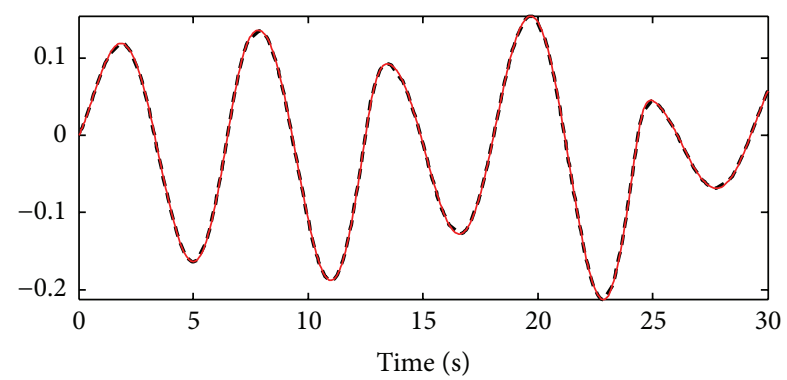

(b)

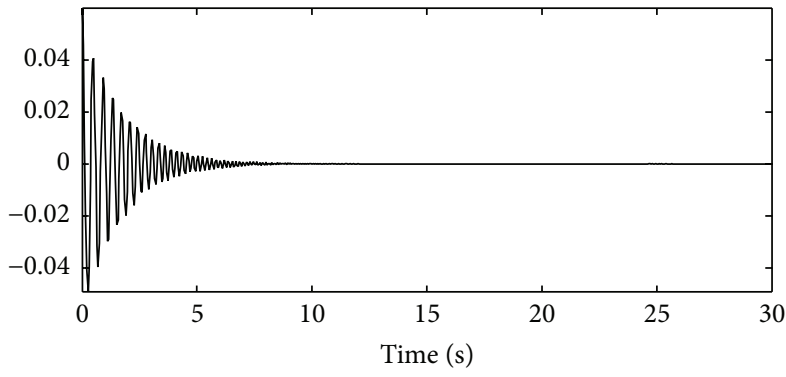

(d)

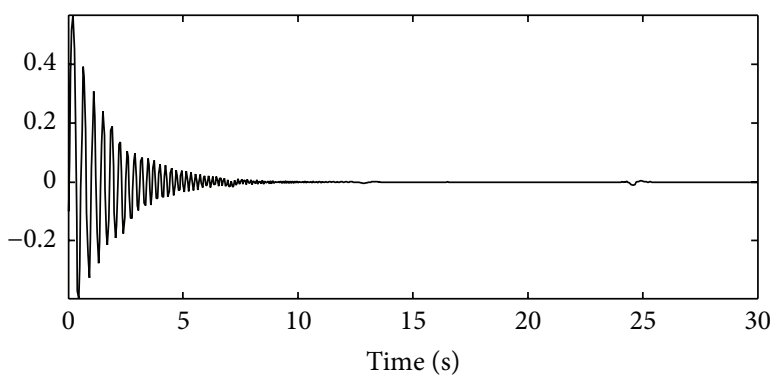

(f)

Figure 2: Behavior of the plant and the observer in $x$ space.

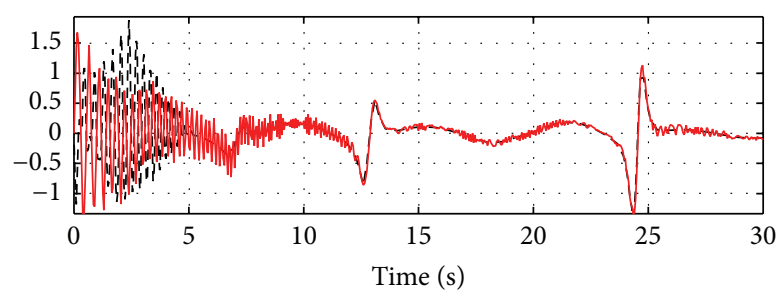

(a)

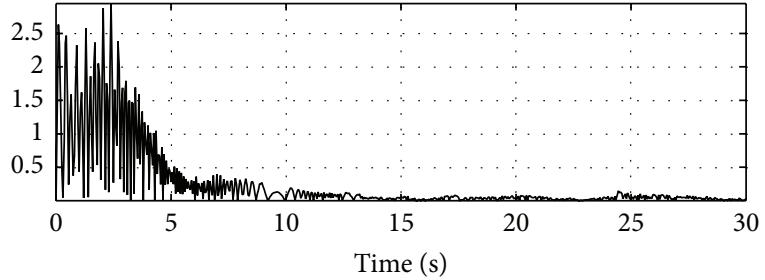

(b)

FIGURE 3: Disturbance and identified disturbance.

seconds. The gains of the controller are $k_{p}=400$ and $k_{v}=10$ and the reference signal is $y_{\text {ref }}(t)=0.4 \sin (0.15 t)$.

In order to observe the effect of disturbance compensation, in the first twenty seconds of the experiments, the control input does not include the disturbance identified; after this time, it is incorporated. The experimental results are shown in Figures 5-10.

Figure 5 shows that the controller estimates properly the angular position because it is identical to the measured angular position; therefore, the estimated velocity, shown in Figure 6, and the disturbances identified, shown in Figure 7, are very close to the actual velocity and disturbance, respectively.

Now, we analyze the closed loop performance. In Figure 8 , we can see the control signal; it has a small change at $t=20 \mathrm{sec}$ when the estimated disturbance is incorporated.

Finally, Figures 9 and 10 show that, in the first 20 seconds, when the disturbances are not compensated, the tracking 


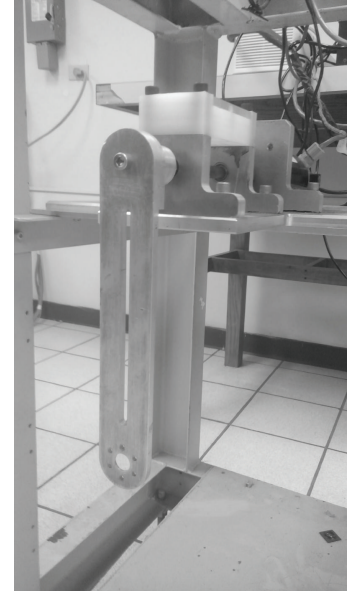

FIGURE 4: The pendulum used in the experiments.

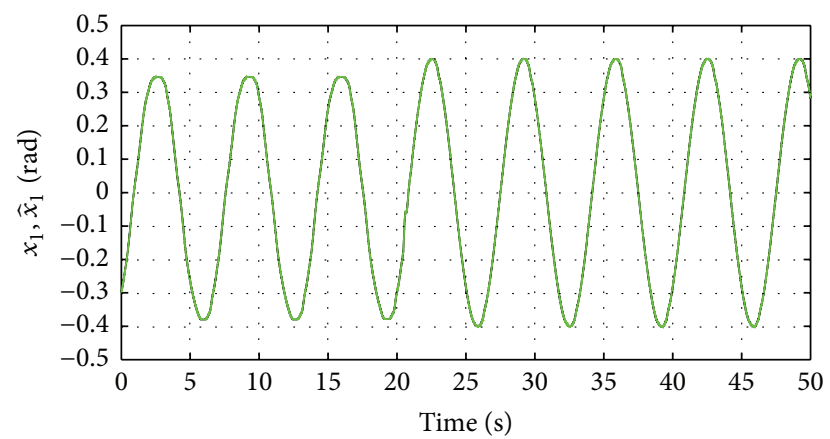

Figure 5: Measured angular position $x_{1}$ and observed angular position $\hat{x}_{1}$.

error is large. When this term is compensated, after 20 seconds, the tracking error decreases considerably in both cases.

\section{Conclusions}

This paper presents the design of a robust observer for a class of SISO nonlinear systems with full relative degree. This observer guarantees, analytically, the asymptotic convergence to the state of the plant in spite of the parametric uncertainties and bounded external disturbances. In addition, the observer identifies the disturbances in the plant without the use of an additional low-pass filter. This observer can be extended directly to other types of multiple input multiple output (MIMO) systems such as mechanical systems modeled by Lagrange equations and to those that can be brought to a normal form.

The performance of the observer has been illustrated in a particular control system but it is possible to make a generalization of this application to mechanical systems and other kinds of dynamic systems. Moreover, the example of the observer for the Rössler system opens the possibility to use the observer for the synchronization of chaotic systems.

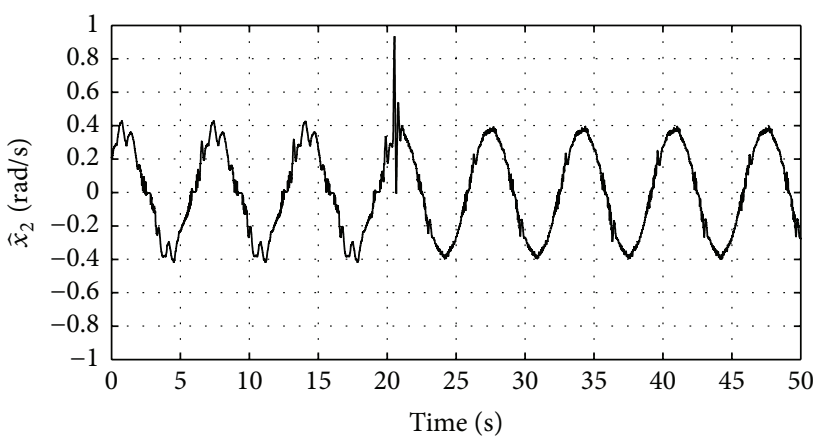

Figure 6: Estimated velocity $\widehat{x}_{2}$.

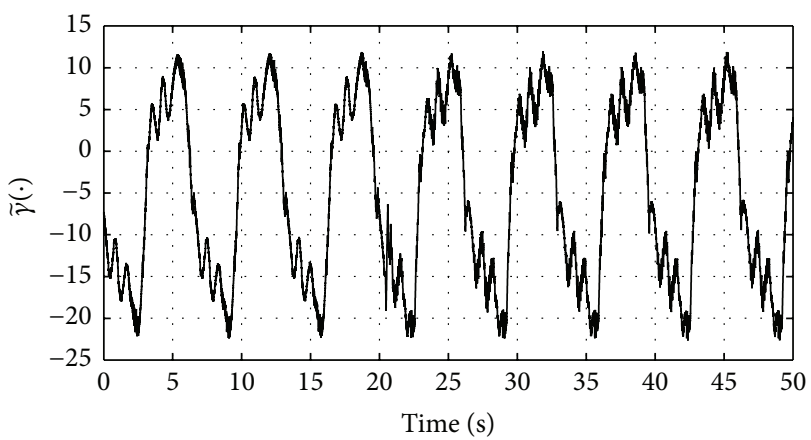

FIGURE 7: Estimated disturbances $\widetilde{\gamma}(\cdot)$.

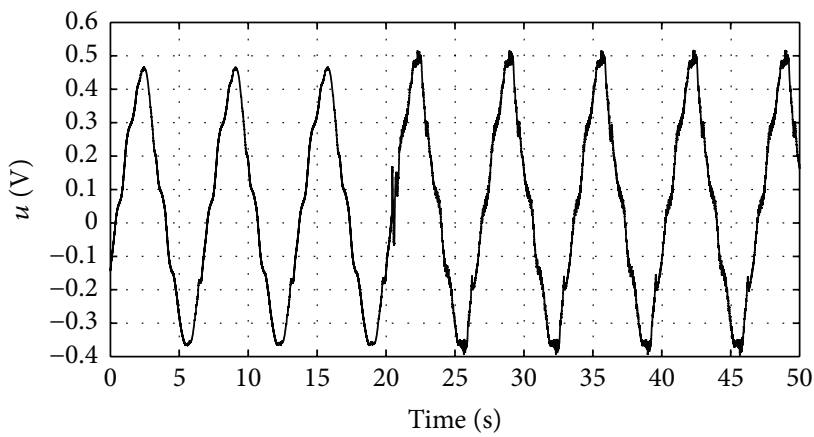

Figure 8: Control input $u$.

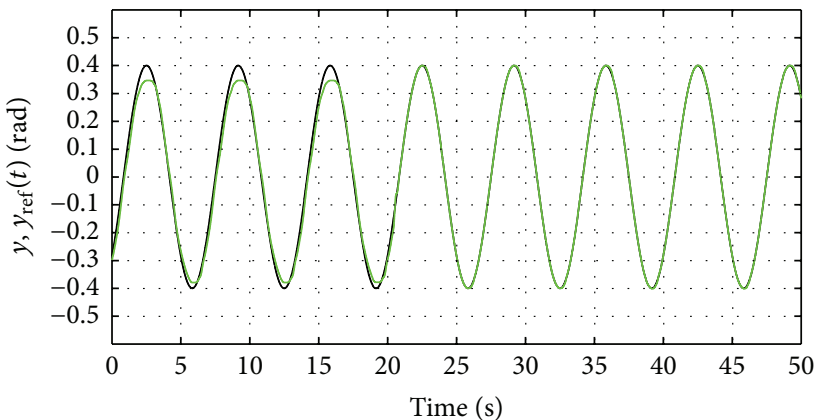

FIGURE 9: Reference signal $y_{\text {ref }}(t)$ (black) and the system output $y$ (green). 


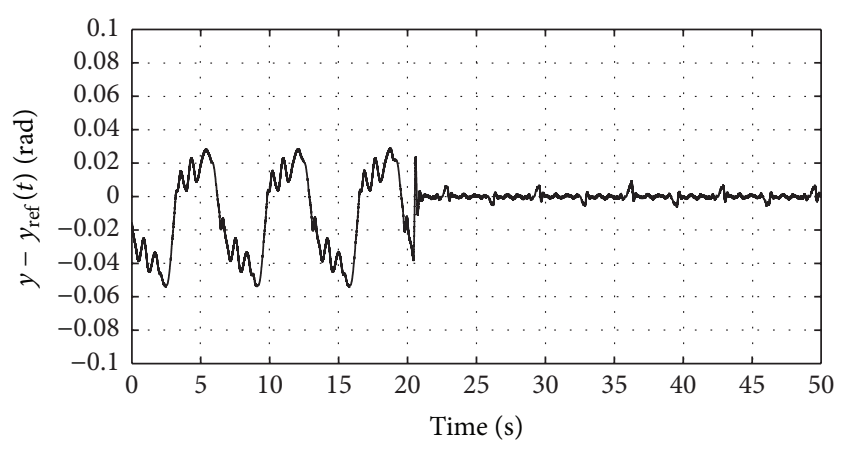

FIGURE 10: Tracking error.

The observer also presents certain practical problems. The use of several cascading stages can degrade the performance of the final stages due to the accumulation of numerical errors, because the variables recovered at each stage are used in the following stages. In order to minimize this problem, the observer needs to be implemented in real-time systems with small sampling times. Under these conditions, no practical problems occur even in third-order systems.

It is important to note that the experimental results are very similar to those obtained with other more complex control techniques such as $H_{\infty}$ and the sliding mode control, with the advantage of not requiring the solution of the Riccati differential equation online [19], whose solution is needed in $H_{\infty}$ case, and having a smooth control signal, which cannot be done with the standard sliding mode control.

\section{Conflict of Interests}

The authors declare that there is no conflict of interests regarding the publication of this paper.

\section{References}

[1] J. Barbot, M. Djemai, and T. Boukhobza, "Sliding mode observers," in Sliding Mode Control in Engineering, p. 11, CRC Press, Boca Raton, Fla, USA, 2002.

[2] G. Besancon, Nonlinear Observers and Applications, Springer, 2007.

[3] X. Chen, T. Fukuda, and K. D. Young, "A new nonlinear robust disturbance observer," Systems \& Control Letters, vol. 41, no. 3, pp. 189-199, 2000.

[4] J. Davila, L. Fridman, and A. Levant, "Second-order slidingmode observer for mechanical systems," IEEE Transactions on Automatic Control, vol. 50, no. 11, pp. 1785-1789, 2005.

[5] Y. C. Huang, H. Y. Li, J. Zhang, and X. Du, "Mars atmospheric entry guidance design by sliding mode disturbance observerbased control," Procedia Engineering, vol. 99, pp. 1062-1075, 2015.

[6] S. Mondal and C. Mahanta, "Adaptive integral higher order sliding mode controller for uncertain systems," Journal of Control Theory and Applications, vol. 11, no. 1, pp. 61-68, 2013.

[7] D. I. Rosas Almeida, J. Alvarez, and L. Fridman, "Robust observation and identification of $n$ DOF Lagrangian systems," International Journal of Robust and Nonlinear Control, vol. 17, no. 9, pp. 842-861, 2007.
[8] K. C. Veluvolu and D. Lee, "Sliding mode high-gain observers for a class of uncertain nonlinear systems," Applied Mathematics Letters, vol. 24, no. 3, pp. 329-334, 2011.

[9] Q. Hu, B. Li, and J. Qi, "Disturbance observer based finite-time attitude control for rigid spacecraft under input saturation," Aerospace Science and Technology, vol. 39, pp. 13-21, 2014.

[10] F. Li, G. Zhao, and J. Huang, "Exact state estimation for linear systems with unbounded disturbances," Circuits, Systems, and Signal Processing, vol. 34, no. 5, pp. 1519-1533, 2015.

[11] J. Yang, F. Zhu, and W. Zhang, "Sliding-mode observers for nonlinear systems with unknown inputs and measurement noise," International Journal of Control, Automation and Systems, vol. 11, no. 5, pp. 903-910, 2013.

[12] A. Levant, "Robust exact differentiation via sliding mode technique," Automatica, vol. 34, no. 3, pp. 379-384, 1998.

[13] J. Davila, L. Fridman, and A. Poznyak, "Observation and identification of mechanical systems via second order sliding modes," International Journal of Control, vol. 79, no. 10, pp. 12511262, 2006.

[14] V. I. Utkin, Sliding Modes in Control and Optimization, vol. 116, Springer, Berlin, Germany, 1992.

[15] J. Alvarez, D. Rosas, and J. Peña, "Analog implementation of a robust control strategy for mechanical systems," IEEE Transactions on Industrial Electronics, vol. 56, no. 9, pp. 3377$3385,2009$.

[16] C. P. Tan and C. Edwards, "Robust fault reconstruction using multiple sliding mode observers in cascade: development and design," in Proceedings of the American Control Conference (ACC '09), pp. 3411-3416, IEEE, St. Louis, Mo, USA, June 2009.

[17] H. K. Khalil and J. W. Grizzle, Nonlinear Systems, vol. 3, Prentice Hall, New Jersey, NJ, USA, 1996.

[18] E. Mosekilde, Y. Maistrenko, and D. Postnov, Chaotic Synchronization: Applications to Living Systems, World Scientific, Singapore, 2002.

[19] A. Isidori and A. Astolfi, "Disturbance attenuation and $H_{\infty}$ control via measurement feedback in nonlinear systems," IEEE Transactions on Automatic Control, vol. 37, no. 9, pp. 1283-1293, 1992. 


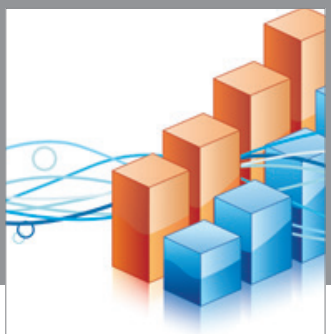

Advances in

Operations Research

vatem alat4

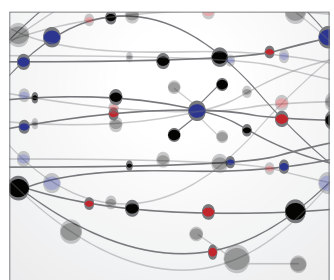

\section{The Scientific} World Journal
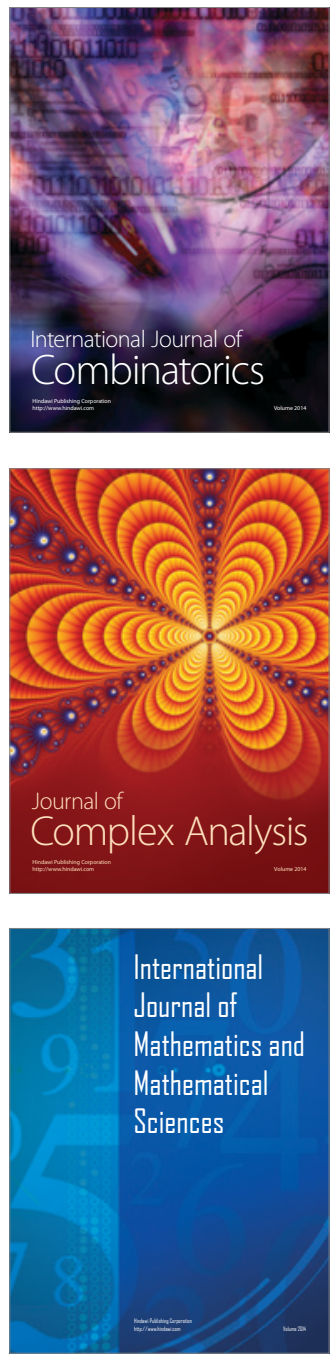
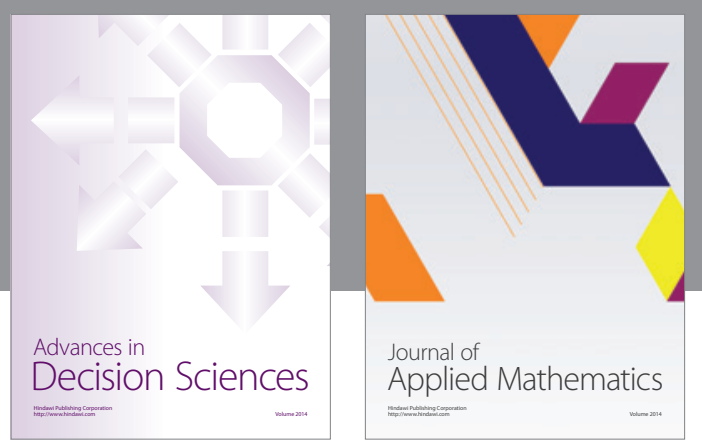

Algebra

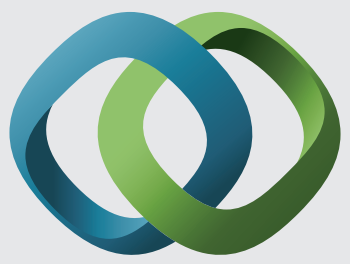

\section{Hindawi}

Submit your manuscripts at

http://www.hindawi.com
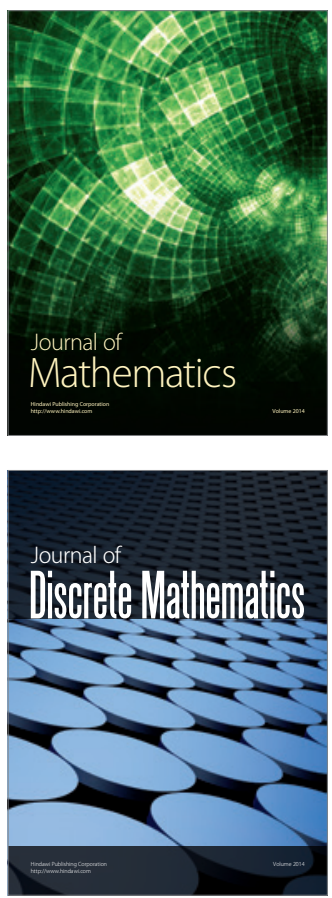

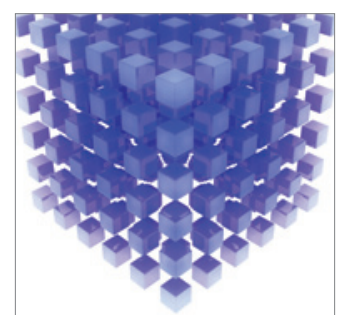

Mathematical Problems in Engineering
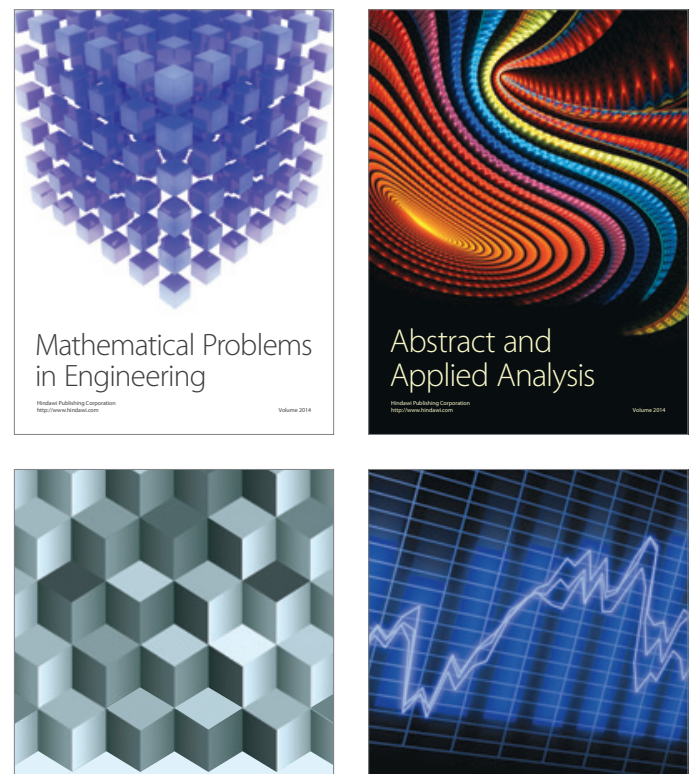

Journal of

Function Spaces

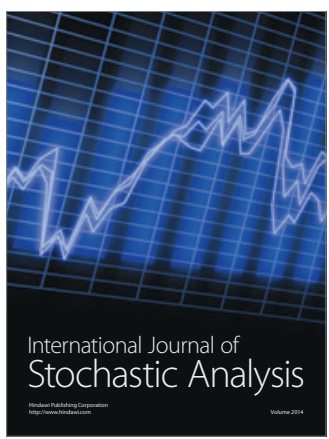

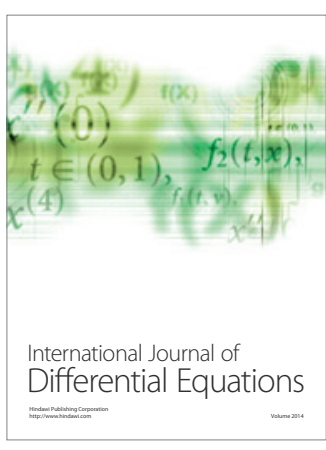
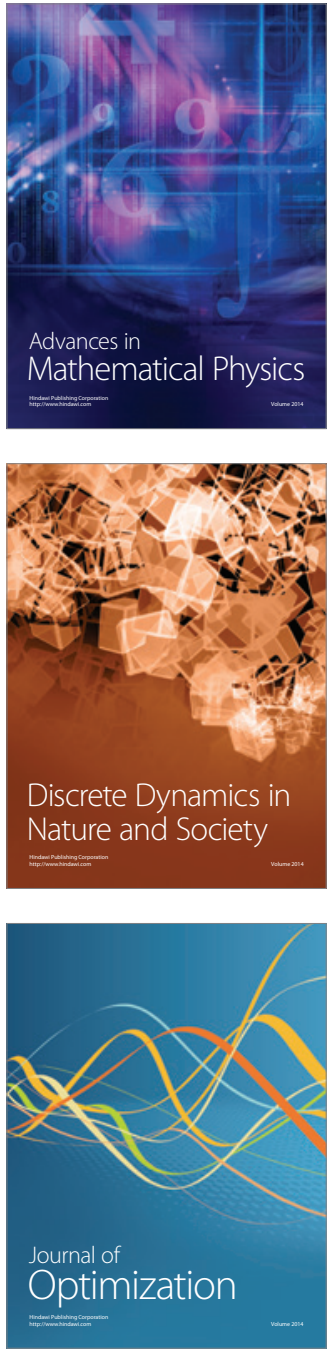